Speirits, Fiona C., Lavery, Martin P. J., Padgett, Miles J., and Barnett, Stephen M. (2014) Optical angular momentum in a rotating frame. Optics Letters, 39 (10). pp. 2944-2946. ISSN 0146-9592

Copyright (C) 2014 Optical Society of America

A copy can be downloaded for personal non-commercial research or study, without prior permission or charge

Content must not be changed in any way or reproduced in any format or medium without the formal permission of the copyright holder(s)

When referring to this work, full bibliographic details must be given

$\underline{\text { http://eprints.gla.ac.uk/93488 }}$

Deposited on: 12 May 2014

Enlighten - Research publications by members of the University of Glasgow http://eprints.gla.ac.uk 


\title{
Optical angular momentum in a rotating frame
}

\author{
Fiona C. Speirits, ${ }^{*}$ Martin P. J. Lavery, Miles J. Padgett, and Stephen M. Barnett \\ School of Physics and Astronomy, University of Glasgow, Glasgow G12 8QQ, UK \\ ${ }^{*}$ Corresponding author: fiona.speirits@glasgow.ac.uk
}

Received February 5, 2014; revised March 26, 2014; accepted March 26, 2014; posted April 9, 2014 (Doc. ID 205993); published May 9, 2014

\begin{abstract}
It is well established that light carrying orbital angular momentum (OAM) can be used to induce a mechanical torque causing an object to spin. We consider the complementary scenario: will an observer spinning relative to the beam axis measure a change in OAM as a result of their rotational velocity? Remarkably, although a linear Doppler shift changes the linear momentum of a photon, the angular Doppler shift induces no change in the angular momentum. Further, we examine the rotational Doppler shift in frequency imparted to the incident light due to the relative motion of the beam with respect to the observer and consider what must happen to the measured wavelength if the speed of light $c$ is to remain constant. We show specifically that the OAM of the incident beam is not affected by the rotating observer and that the measured wavelength is shifted by a factor equal and opposite to that of the frequency shift induced by the rotational Doppler effect. @ 2014 Optical Society of America

OCIS codes: (080.4865) Optical vortices; (070.2580) Paraxial wave optics.

http://dx.doi.org/10.1364/OL.39.002944
\end{abstract}

The angular momentum of light has been studied since Poynting first considered a circularly polarized beam by analogy with the mechanical properties of a revolving shaft in 1909 [1]. The rotation of the electric and magnetic field vectors around the axis of propagation, which results in the circular polarization of light, has afforded both theoreticians and experimentalists a plethora of opportunities for study for many years [2]. Careful consideration of the spin angular momentum per photon associated with the polarization reveals a shortfall: there is a second contribution in the form of an orbital angular momentum (OAM), such that the total angular momentum of light is the sum of the spin and orbital momenta [3]:

$$
\boldsymbol{J}=\boldsymbol{S}+\boldsymbol{L} .
$$

These spin $S$ and orbital $L$ components of the total angular momentum can be manipulated and utilized independently of each other and much study has been made of the effect of frame rotation on both the spin angular momentum of light $[\underline{4}, \underline{5}]$ and the Hamiltonian of matter waves in Bose-Einstein condensates [6]. Frame rotation has also been employed when considering reflection and interference patterns [7-9]. However, the effect of frame rotation on the OAM of light itself has yet to be fully explored. Recent experiments suggest that the measured OAM of a light beam will not be altered by the rotational motion of an observer [10]. In this Letter, we address the theoretical basis of this, showing why the OAM does not change. In addition to this, we also consider what happens to the wavelength of a beam of light as measured by a rotating observer. Intuitively, one must expect a shift in order to counterbalance the rotational Doppler shift in frequency $[\underline{11}, 12]$, as no change in the wavelength would suggest a contradiction to the constancy of the speed of light. We confirm that this is indeed the case and present the mechanism responsible for the wavelength change.

A Bessel beam propagating in the positive $z$ direction is expressed in standard cylindrical coordinates as

$$
\psi=J_{\ell}(\kappa \rho) e^{i k z} e^{i \ell \phi} e^{-i \omega t},
$$

where $\ell \hbar$ is the OAM per photon []ㅡ and $\kappa^{2}+k^{2}=\omega^{2} / c^{2}$. Here we consider the familiar paraxial regime in which $\kappa^{2} \ll k^{2}$ so that $k \simeq 2 \pi / \lambda$, where $\lambda$ is the wavelength. We consider an observer rotating around the beam axis in the $x-y$ plane and ask what they will measure due to their motion relative to the beam. We assume a rotation rate

$$
\frac{\Omega}{\kappa} \ll c,
$$

so that an observer rotating at a radius where the field is a maximum is moving non-relativistically. Let us define primed and unprimed coordinates for the stationary $S$ and rotating $S^{\prime}$ reference frames, respectively. For an observer rotating at angular velocity $\Omega$, their azimuthal coordinates in the two frames, $\phi$ and $\phi^{\prime}$, are related by

$$
\phi^{\prime}=\phi-\Omega t .
$$

We can therefore transform Eq. (2) to the observer's reference frame to obtain

$$
\psi=J_{\ell}(\kappa \rho) e^{i k z} e^{i \ell\left(\phi^{\prime}+\Omega t\right)} e^{-i \omega t},
$$

and hence

$$
\psi=J_{\ell}(\kappa \rho) e^{i k z} e^{i \ell \phi^{\prime}} e^{-i(\omega t-\ell \Omega t)},
$$

where the rotational Doppler shift in frequency appears in the final exponential with the expected dependence on $\ell$ that is $\Delta \omega=\omega \ell$ [13].

We can now derive the essential properties of the beam and consider the effect of this rotational motion; we begin with the OAM. The angular momentum eigenfunctions $\psi_{\ell}(\rho, \phi)$, written in standard cylindrical coordinates, satisfy

$$
-i \hbar \frac{\partial}{\partial \phi} \psi_{\ell}(\rho, \phi)=\hbar \ell \psi_{\ell}(\rho, \phi)
$$

Transforming this to the rotating observer's frame changes only the $\phi$ coordinate: 


$$
-i \hbar \frac{\partial}{\partial \phi^{\prime}} \psi_{\ell}\left(\rho, \phi^{\prime}\right)=\hbar \ell \psi_{\ell}\left(\rho, \phi^{\prime}\right)
$$

It is evident, therefore, that the rotating observer will measure the same value for $\ell$ as if they were stationary with respect to the beam. This OAM invariance is an inevitable consequence of the fact that a rotationally symmetric field has the same rotational symmetry when viewed from a rotating frame. Hence $\ell$ is the same integer in both frames and the OAM will not change for a rotating observer. One can also think of $\ell$ as the number of discreet phase steps in the cross-section of the beam at a single snapshot in time. There is no mechanism by which this can change solely due to the motion of the observer.

Having established the invariance of the OAM due to rotation, it is of interest to consider the effect of this rotation on the wavelength and frequency of the beam. As may been seen from Eq. (5), the frequency in the rotating reference frame $\omega^{\prime}$ is not equal to $\omega$, a consequence of the rotational Doppler shift. Yet as the term associated with the axial propagation of the wavevector, $e^{i k z}$, appears unchanged, it seems that the wavelength is also unaffected by the observer's motion and hence $\omega^{\prime} k^{\prime} \neq c$. The resolution of this anomaly lies in the fact that the wavelength $\lambda$ is determined by how far along the beam one must travel $\Delta z$ from an initial point $(\rho, \phi, z)$ to reach the next point with the same phase at $(\rho, \phi, z+\Delta z)$, as shown in Fig. 1. However, for a rotating observer these two loci do not have the same $\phi$ coordinate, as can be seen in Fig. 2 . This retarded position must be accounted for, akin to synchronizing the respective frames' clocks due to the finite value of $c$. As there are two reference frames and hence

a)

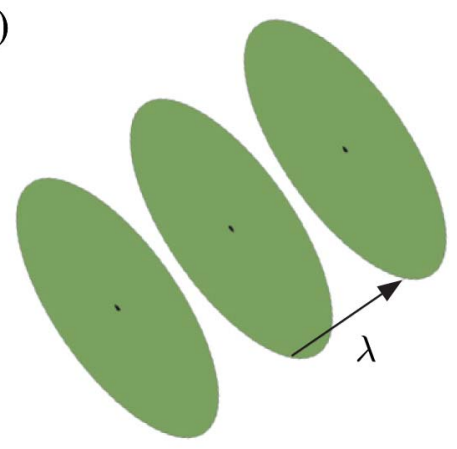

b)

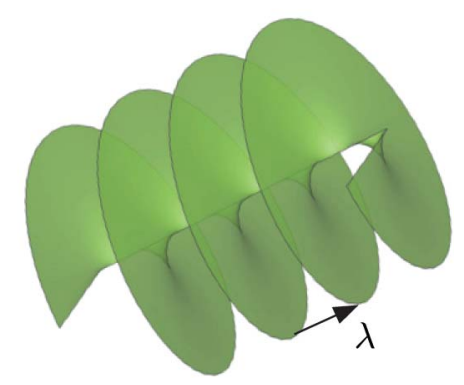

Fig. 1. Wavelength $\lambda$ is defined as the distance along the beam between two points with the same phase, shown for (a) a Gaussian beam with flat phase fronts and (b) an $\ell=1$ beam with helical phase fronts. a)

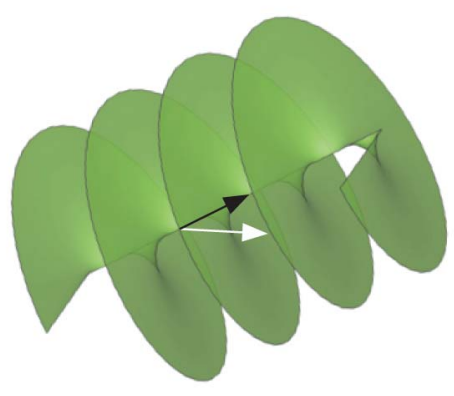

b)

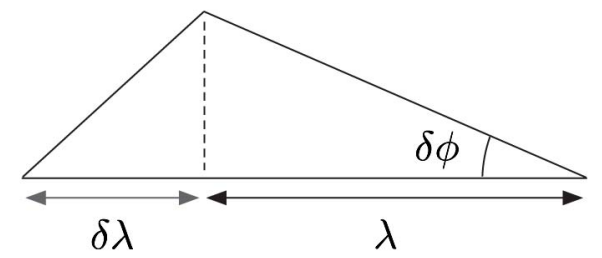

Fig. 2. Change in $\phi$ coordinate from $\phi$ (black arrow) to $\phi^{\prime}$ (white arrow) due to the rotation of the observer can be seen in (a), while the corresponding relative change in wavelength $\delta \lambda$ is shown in (b).

two descriptions of this problem, it is illustrative to consider it from both perspectives.

In the laboratory rest frame (a stationary beam and a rotating observer) the relative change in distance, and hence wavelength, is related to the change in angular position due to the observer's rotation, illustrated in Fig. 2:

$$
k_{z} \delta z=\ell \delta \phi
$$

As $\delta \phi=\Omega t$ and $\lambda=2 \pi / k_{z}$ the fractional change in wavelength can therefore be expressed as

$$
\frac{\delta \lambda}{\lambda}=\frac{\ell \Omega}{c k_{z}} \simeq \frac{\ell \Omega}{\omega}
$$

This can be written as a fractional change in $k_{z}$

$$
\delta k=-\frac{\ell \Omega}{\omega} k
$$

and therefore the wavenumber in the rotating frame is

$$
k^{\prime}=k\left(1-\frac{\ell \Omega}{\omega}\right) \text {. }
$$

From Eq. (3), the frequency shift is

$$
\omega^{\prime}=\omega-\ell \Omega=\omega\left(1-\frac{\ell \Omega}{\omega}\right) .
$$

Hence, both $\omega$ and $k_{z}$ are changed by the same factor, thus 


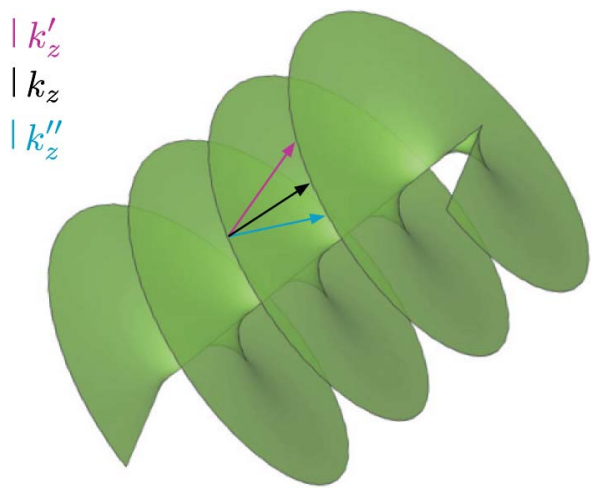

Fig. 3. Effect of an observer's rotation around the beam axis can be seen to either increase, $k_{z}^{\prime \prime}$, or decrease, $k_{z}^{\prime}$, the measured wavelength, compared with the wavelength in the non-rotating case $k_{z}$.

$$
\frac{\omega^{\prime}}{k^{\prime}}=\frac{\omega}{k}=c .
$$

Rotating into the helical phase fronts will reduce the measured wavelength, while rotating in the opposite direction will increase the observed wavelength, as seen in Fig. 3. Conceptually, this is akin to walking down a helical staircase: by leaning forward (equivalent to rotating into the beam), the distance to the stairs above is shortened and one runs the risk of hitting one's head. Leaning backward, however, increases the gap to the stairs above and allows a taller person to avoid a collision. Inverting the sign of the beam OAM number reverses the sense of rotation such that the observer is now walking up the staircase. Leaning forward offers more headroom while leaning backward may result in a nasty bump.

The rotational frequency shift can also be viewed in the complementary frame $S^{\prime}$ of a stationary observer viewing a rotating beam. In this case we require the interval between points of the same phase (one wavelength) to be $\Delta z^{\prime}=c \Delta t^{\prime}$. From Eq. (5) this provides the constraint that

$$
e^{i k \Delta z^{\prime}} e^{i \ell \Delta \phi^{\prime}}=1
$$

It is evident that either $\left(\Delta z^{\prime}=0, \Delta \phi^{\prime}=0\right)$ or

$$
k \Delta z^{\prime}+\ell \Delta \phi^{\prime}=2 \pi
$$

for a single wavelength.

In the unprimed lab frame we know that $\Delta z=c \Delta t$ and $k \Delta z+\ell \Delta \phi=2 \pi$. Therefore, combining these with Eq. (3) we obtain

$$
\Delta z^{\prime}=\Delta z\left(1+\frac{\ell \Omega}{c k}\right)
$$

This again corresponds to the wavenumber in the rotating frame being shifted by the same factor as the frequency

$$
k^{\prime}=k\left(1-\frac{\ell \Omega}{c k}\right)
$$

thus again maintaining the invariance of $c$.

The phenomenon described in this Letter is distinct from the more familiar transformation to a uniformly moving frame for which the Lorentz transformation combines the coordinate in the direction of motion with time. The Doppler frequency shift and the associated change of wavevector then arise naturally from the fact that the phase of the field is a Lorentz scalar. For rotational motion however, the constituent plane waves forming our beam do not have an azimuthal component [14] and the requisite change in wavevector instead appears in the z-component as we have demonstrated.

We have shown that an observer rotating in the plane perpendicular to the axis of a beam carrying OAM will measure no change in the OAM number $\ell$, which is consistent with experimental observation. This invariance of the angular momentum under rotation is in direct contrast to the change in linear momentum that occurs in the linear Doppler effect. In addition to this we have demonstrated that the expected shift in frequency due to the rotational Doppler effect is exactly balanced by a change in the wavelength as measured by the rotating observer, resulting in no contradiction to the invariance of the speed of light.

This work was supported by the UK EPSRC and the Defense Advanced Research Projects Agency InPho program through U.S. Army Research Office award W911NF10-1-0395. M.J.P. thanks the Royal Society and Wolfson Foundation.

\section{References}

1. J. H. Poynting, Proc. R. Soc. London A 82, 560 (1909).

2. W. A. Shurcliff, Polarized Light: Production and Use (Harvard, 1962).

3. L. Allen, M. W. Beijersbergen, R. J. C. Spreeuw, and J. P. Woerdman, Phys. Rev. A 45, 8185 (1992).

4. B. A. Garetz, J. Opt. Soc. Am. 71, 609 (1981).

5. G. Nienhuis, Opt. Commun. 132, 8 (1996).

6. J. Javanainen, S. M. Paik, and S. M. Yoo, Phys. Rev. A 58, 580 (1998).

7. A. Y. Okulov, J. Opt. Soc. Am. B 29, 714 (2012).

8. M. P. MacDonald, K. Volke-Sepulveda, L. Paterson, J. Arlt, W. Sibbett, and K. Dholakia, Opt. Commun. 201, 21 (2002).

9. J. Arlt, M. P. MacDonald, L. Paterson, W. Sibbett, K. Volke-Sepulveda, and K. Dholakia, Opt. Express 10, 844 (2002).

10. M. P. J. Lavery, F. C. Speirits, S. M. Barnett, and M. J. Padgett, Science 341, 537 (2013).

11. I. Bialynicki-Birula and Z. Bialynicka-Birula, Phys. Rev. Lett. 78, 2539 (1997).

12. M. V. Vasnetsov, V. A. Pas'ko, and M. S. Soskin, Pis'ma v Zh. Èksper. Teoret. Fiz. 81, 699 (2005).

13. J. Courtial, D. A. Robertson, K. Dholakia, L. Allen, and M. J. Padgett, Phys. Rev. Lett. 81, 4828 (1998).

14. F. C. Speirits and S. M. Barnett, Phys. Rev. Lett. 111, 103602 (2013). 